\title{
Molecularly Imprinted Micro-Solid-Phase Extraction for the Selective Determination of Phenolic Compounds in Environmental Water Samples with High Performance Liquid Chromatraphy
}

\author{
Qinzhong Feng $^{1^{*}, \text { Yang Chen }}{ }^{1}$, Diandou Xu ${ }^{1}$, Liyuan Liu ${ }^{1}$, Zhengjie Zhang ${ }^{2}$ \\ ${ }^{1}$ The National Center for Nano-Science and Technology Laboratory for Bio-Environmental Effects of \\ Nanomaterials and Nanosafety, Institute of High Energy Physics, Chinese Academy of Sciences, Beijing, China \\ ${ }^{2}$ Shenyang Academy of Environmental Sciences, Shenyang, China \\ Email: *fengqzh@ihep.ac.cn
}

Received May 8, 2013; revised July 3, 2013; accepted July 10, 2013

Copyright (C) 2013 Qinzhong Feng et al. This is an open access article distributed under the Creative Commons Attribution License, which permits unrestricted use, distribution, and reproduction in any medium, provided the original work is properly cited.

\begin{abstract}
2,4,6-trichlorophenol molecularly imprinted suspension polymer has been prepared and applied to the molecularly imprinted micro-solid-phase extraction procedure for selective preconcentration of phenolic compounds from environmental water samples. The influence of functional monomer, cross-linker, polymerization condition, porogen, and the ratio of template molecule and functional monomer to cross-linker on the size of the obtained particles were investigated. It was found that methyacrylic acid as functional monomer, divinylbenzene as cross-linker, the molar ratio of template molecule and functional monomer to cross-linker was 1:4:20, the amount of AIBN was $100 \mathrm{mg}$, ultraviolet radiation at $365 \mathrm{~nm}$ were the optimal conditions, and at these conditions, the polymers had the best adsorption efficiency and had the monodispersity of $2-3 \mu \mathrm{m}$ microsphere particles. The characteristics of the MIMSPE method were valid by high performance liquid chromatography. This MIMSPE-HPLC method has been successfully applied to the direct preconcentration and determination of phenolic compounds (phenol, 4-chlorophenol, 2,4-dichlorophenol, 2,4,6trichlorophenol, pentachlorophenol) in environmental water samples.
\end{abstract}

Keywords: Suspension Polymerization; Molecularly Imprinted Micro-Solid-Phase Extraction; 2,4,6-Trichlorophenol; Phenolic Compounds; High Performance Liquid Chromatography

\section{Introduction}

Molecularly imprinted polymers (MIPs) have attracted considerable research interests due to their predetermined selectivity toward a given analyte or a group of structurally related species. Typically, MIPs are prepared in the non-covalent approach, hydrogen bonds and other polar interaction between template molecules and functional monomers. The classical way of synthesizing MIPs is by bulk polymerization following tedious time- and laborintensive protocols. The procedure involves crushing, grinding and sieving these resultant monoliths to an appropriate particle size for the particular studies which will be undertaken [1]. These irregular particles generally give less efficient column packing for chromatography and often prove troublesome in chromatography process,

*Corresponding author. therefore, when MIPs are used as affinity-based chromatography media, HPLC stationary phases or solid-phase extraction (SPE) media, it is desirable to prepare the spherical and mono-dispersed beads.

Bead polymers can be prepared by dispersion polymerization [2], by precipitation polymerization [3], or by core-shell polymerization method [4-6]. Alternatively, beaded MIPs may be obtained via the use of grafting procedures where one distinguishes between "grafting to" and "grafting from" techniques [7], the latter referring to polymerization initiated from a preformed polymer or from the surface of a solid support. However, these techniques suffer from the presence of water or highly polar organic solvents during the polymerization procedure, which frequently decreases specific interactions between functional monomers and template molecules in most commonly applied non-covalent imprinting 
mixtures. Hence, for the synthesis of molecularly imprinted microspheres while retaining high selectivity along with control on particle shape and size, a synthetic route avoiding the use of dispersants such as water or polar solvents is demanded. Suspension techniques have been applied for the preparation of imprinted nanospheres for a variety of different analytes and applications $[8,9]$.

Typically, water is used as a continuous phase to suspend a droplet of pre-polymerization mixtures in the presence of a stabilizer or surfactant when spherical MIPs are prepared by conventional suspension polymerization [10]. However, water is not suitable for the synthesis of MIPs relying on hydrogen bonds since water will interfere with the interactions between functional monomers and printing molecules required for the recognition process during molecular imprinting. Recently, two new suspension polymerization techniques based on droplets of pre-polymerization mixtures formed in liquid perfluorocarbon $[11,12]$ or mineral oil (liquid paraffin) $[13,14]$ have been developed. These liquids were used as a continuous phase instead of water. It is interesting that no stabilizers or surfactants were required for the droplet formation. Liquid perfluorocarbons are immiscible with almost all organic solvents, and that many combinations of monomers and crosslinkers and a wide range of solvents, employed for suspension polymerization, could be utilized. However, it is required to synthesize perfluoro polymeric surfactants. The mineral oil consists of a mixture of aliphatic hydrocarbons and it is very easy to comprise the formation of droplets of prepolymerization mixtures directly in mineral oil by vigorous mixing followed by transformation of the droplets into solid spherical beads by photo polymerization or by heat polymerization.

2,4,6-trichlorophenol $(2,4,6-\mathrm{TCP})$ is used as an antiseptic, a pesticide for wood, leather, and glue preservation, and as an anti-mildew treatment. Due to its high toxicity, carcinogenic properties, and persistence in the environment $[15,16]$, much attention has been focused on developing cost-effective analytical methods for determining these compounds in environmental samples at low concentration levels. Nowadays, non-covalent molecularly imprinted polymers (MIPs) have increasingly been developed as mimics of natural molecular receptors. However, to our best knowledge, there is no yet a report for preparing 2,4,6-TCP imprinted micro- sphere polymers by suspension polymerization method which could be used as SPE sorbent for the separation analytes from complex matrices.

Micro-solid phase extraction is a novel and feasible alternative to multistep SPE method for preconcentration the analytes in complex samples. In 2006, Basheer et al. [17] firstly reported this approach in which multiwalled carbon nanotube (MWCNTs) was used to extract organophosphorus pesticides (OPPs) from environmental samples. Like SPE, this extraction procedure involved analyte adsorption followed by solvent desorption but on a much smaller scale. The extraction device is easy to prepare in-house at reasonable cost. This technique is robust and durable and a single device can be used for 30 extractions [18].

In this work, 2,4,6-trichlorophenol (2,4,6-TCP) molecularly imprinted polymers were prepared by suspension polymerization method using 2,4,6-TCP as template molecule, liquid papaffin oil as non-aqueous media, respecttively. The factors, which will affect the affinity efficiency, such as the monomers, the crosslinkers, polymerization conditions, were investigated. A novel extraction technique, MIMSPE, involving the use of microsphere molecularly imprinted polymer wrapped in a porous membrane sheet, was used for selective preconcentration of phenolic compounds and the concentrated phenolic compounds were analyzed with HPLC. The method was validated and used to determine phenolic compounds from environmental water samples with satisfactory results.

\section{Experimental}

\subsection{Reagents and Apparatus}

2,4,6-TCP, 4-vinylpyridine (4-VP), methyacrylic acid (MAA), and ethylene glycol dimethacrylate (EGDMA) were all from Acros Organics; trimethylolpropane trimethacrylate (TRIM) and divinylbenzene (DVB) were from Sigma-Aldrich Chemie. 2,2'-Azobisisobutyronitrile (AIBN) was from Shanghai No.4 Reagent and H.V. Chemical Company (Shanghai China), and acetonitrile was from J. T. Baker (USA). The monomers were purified by standard procedures to remove stabilizers. AIBN was recrystallized from ethanol. Acetic acid and chloroform were purchased from Beijing Chemical Reagent Company (Beijing, China); methanol, ethanol, and toluene were from Beijing Bei Hua Finachemical Company (Beijing, China). Polypropylene membranes (pore size: $0.15 \mu \mathrm{m}, 0.22 \mu \mathrm{m}$ ), nylon membranes (pore size: 0.15 $\mu \mathrm{m})$ and fibre membranes (pore size: $0.15 \mu \mathrm{m}$ ) were purchased from Shenghemo Company (Beijing, China). Mineral oil (white heavy) was from Aldrich (Milwaukee, USA).

The chemicals for HPLC were at least of HPLC grade. Stock standard solutions of phenol, 4-chlorophenol (4CP), 2,4-dichlorophenol (2,4-DCP), 2,4,6-TCP and pentachlorophenol (PCP) were fresh prepared in acetonitrile, and stored at $4^{\circ} \mathrm{C}$ in a refrigerator. All working solutions were prepared by diluting the stock standard solutions with deionized ultrapure water $\left(18.3 \mathrm{M} \Omega \cdot \mathrm{cm}^{-1}\right.$, EasyPure LF Barnstead, USA) to the needed concentra- 
tions correspondingly.

The resulting suspension imprinted polymers were characterized by scanning electron microscope (SEM) (S-3000N, Hitachi, Japan) and by nitrogen adsorption/ desorption analysis of Brunauer-Emmett-Teller (ASAP 2000, Micromeritics, USA).

To evaluate the polymers, these particles were suspended in water, the solutions were determined with UV2401PC spectrophotometer (Shimadzu Corporation, Japan).

\subsection{Preparation of Molecular Imprinted Polymers with Suspension Methods}

For preparation of the imprinted polymers, the template molecule 2,4,6-TCP $(1 \mathrm{mmol})$, the functional monomer MAA or 4-VP, and the cross-linker EGDMA, DVB, or TRIM were dissolved in the porogen following the molar ratios listed in Table 1. After addition of AIBN, the solutions were sonicated for 10 min and deoxygenated with nitrogen for $10 \mathrm{~min} .3 \mathrm{~mL}$ of each mixture was each added to $27 \mathrm{~mL}$ of mineral oil in $50-\mathrm{mL}$ laboratory bottles. The mixtures were dispersed with vortex shaker for $5 \mathrm{~min}$. The bottles were placed under the UV-lump and polymerized at $365 \mathrm{~nm}$ overnight or placed in $60^{\circ} \mathrm{C}$ water bath and polymerized overnight. The beads were filtered and washed with the mixture solution of chloroform and acetone. MIP particles were washed with the mixture of acetonitrile/acetic acid (acetonitrile:acetic acid $=90: 10$, $\mathrm{v} / \mathrm{v}$ ) in a soxhlet extraction. Finally, the particles were dried in a vacuum at $35^{\circ} \mathrm{C}$ overnight. A corresponding control polymer without 2,4,6-TCP, was prepared in the same procedure (Kempe et al. 2006).

\subsection{Binding Characteristic Assays}

The binding properties of MIP to 2,4,6-TCP were studied by batch method, dynamic method as it were reported before [19]. The amount of 2,4,6-TCP left in the solution after rebinding was detected through UV absorbance. In a typical rebinding experiment, for batch method, $5 \mathrm{~mL}$ of different concentration 2,4,6-TCP was added in 5.0 $\mathrm{mg}$ washed and dried MIPs. Then the suspensions were sealed and were oscillated for $20 \mathrm{~h}$ at room temperature to ensure equilibration. After centrifuged at $3500 \mathrm{rmp}$ for $10 \mathrm{~min}$, the concentration of free 2,4,6-TCP in the supernatant was detected by measuring the absorbance at $\lambda_{\max }=294 \mathrm{~nm}$. The amount of 2,4,6-TCP bound to the polymer was calculated by subtracting the concentration of free 2,4,6-TCP from the initial concentration.

For the dynamic method, $30.0 \mathrm{mg}$ imprinted microsphere polymers $\left(2^{\#}\right)$ was added to $2,4,6-\mathrm{TCP}$ aqueous solution $\left(200 \mathrm{mg} \cdot \mathrm{L}^{-1}, 30.0 \mathrm{~mL}\right)$. The solution was shaken at room temperature. The absorption of the solution at $\lambda_{\max }=294 \mathrm{~nm}$ was determined at $10 \mathrm{~min}, 30 \mathrm{~min}, 2 \mathrm{~h}, 6 \mathrm{~h}$, and $20 \mathrm{~h}$, respectively. The amount of 2,4,6-TCP bound to the polymer was determined by subtracting the concentration of free 2,4,6-TCP from the initial concentration with the UV-2401PC.

\subsection{Preparation of the MIMSPE}

The MIMSPE device consists of the MIPs enclosed within a polypropylene flat-sheet membrane envelope which is shown in Figure 1 and the preparation procedure was the same as reported before [20].

\subsection{Determination of 2,4,6-TCP with HPLC}

High performance liquid chromatograph (HPLC, Shima$\mathrm{dzu}$, Japan) for determining the phenolic compounds (phenol, 4-CP, 2,4-DCP, 2,4,6-TCP and PCP) consisted of a binary LC-10ADvp pump, an DGU-12A on-line degasser, a CTO-10Avp column oven, a SPD-10Avp UV-Vis detector and SCL-10Avp system controller. The injection loop volume was $20 \mu \mathrm{L}$, and the analytical column was a $25 \times 0.46 \mathrm{~cm}$ i.d. spherical ODS. The mobile phase consisted of Milli-Q-quality water as solvent

Table 1. Conditions for the synthesis of polymers $\left(R=Q_{\max , \mathrm{MIP}} / Q_{\max , \mathrm{NIP}}\right)$.

\begin{tabular}{cccccccc}
\hline Number $^{\#}$ & Functional monomer & Cross linker & AIBN $(\mathrm{mg})$ & Molar ratio & Polymerization condition & Particle diameter & $\mathrm{R}$ \\
\hline 1 & MAA & DVB & 100 & $1: 4: 20$ & $60^{\circ} \mathrm{C}$ & $300 \mathrm{~nm}$ & 1.14 \\
2 & MAA & DVB & 100 & $1: 4: 20$ & $365 \mathrm{~nm}$ & $2-3 \mu \mathrm{m}$ & 2.34 \\
3 & MAA & EGDMA & 100 & $1: 4: 20$ & $365 \mathrm{~nm}$ & $300 \mathrm{~nm}$ & 1.20 \\
4 & MAA & TRIM & 100 & $1: 4: 20$ & $365 \mathrm{~nm}$ & $300 \mathrm{~nm}$ & 1.16 \\
5 & 4 -VP & DVB & 100 & $1: 4: 20$ & $365 \mathrm{~nm}$ & $500 \mathrm{~nm}$ & 1.11 \\
6 & MAA & DVB & 20 & $1: 4: 20$ & $365 \mathrm{~nm}$ & $2-3 \mu \mathrm{m}$ & 1.10 \\
7 & MAA & DVB & 60 & $1: 4: 20$ & $365 \mathrm{~nm}$ & $2-3 \mu \mathrm{m}$ & 1.17 \\
8 & MAA & DVB & 160 & $1: 4: 20$ & $365 \mathrm{~nm}$ & $500 \mathrm{~nm}$ & 1.13 \\
9 & MAA & DVB & 100 & $1: 4: 10$ & $365 \mathrm{~nm}$ & $3-5 \mu \mathrm{m}$ & 1.30 \\
10 & MAA & DVB & 100 & $1: 4: 30$ & $365 \mathrm{~nm}$ & $500 \mathrm{~nm}$ & 1.03 \\
\hline
\end{tabular}




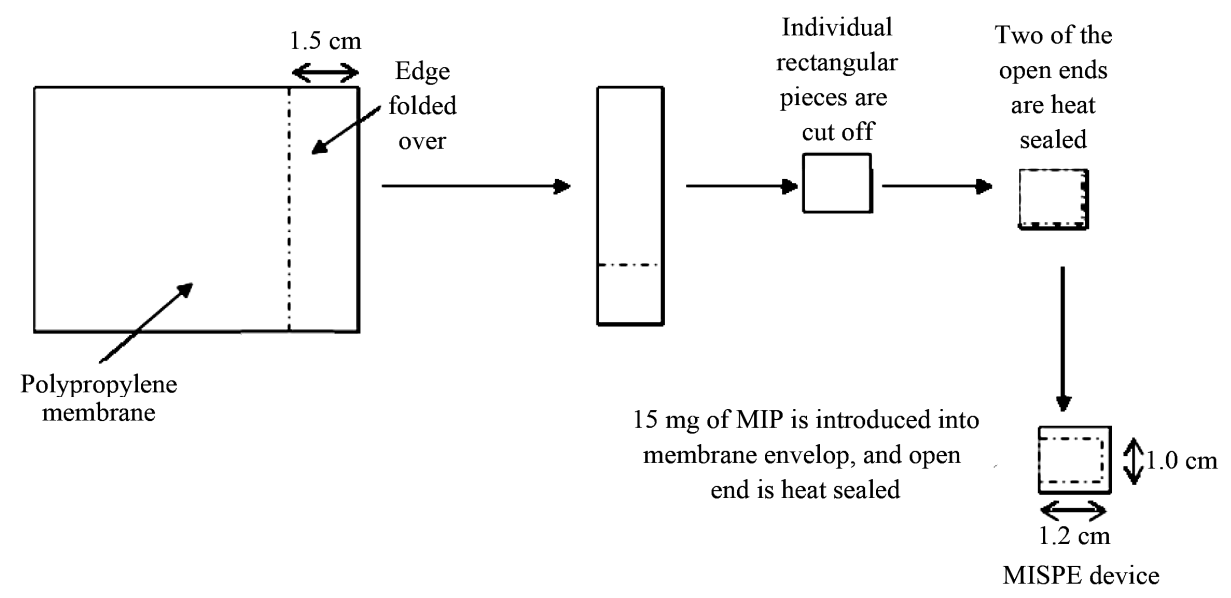

Figure 1. Schematic of preparation of a MIMSPE device.

$\mathrm{A}$ and acetonitrile (containing $0.3 \%(\mathrm{v} / \mathrm{v})$ acetic acid) as solvent B. Both solvent A and solvent B were mixed prior to reaching the analytical column. The flow rate of the mobile phase was $0.5 \mathrm{~mL} \cdot \mathrm{min}^{-1}$, and the gradient profile was $20 \%-40 \%$ B from 0 to $5 \mathrm{~min}, 60 \% \mathrm{~B}$ at 7.5 $\mathrm{min}, 80 \% \mathrm{~B}$ at $20 \mathrm{~min}$, and then isocratic elution for 10 $\min$. The oven temperature was set at $40^{\circ} \mathrm{C}$ then and compounds were detected at $294 \mathrm{~nm}$, except for phenol and 2,4-DCP which were detected at $280 \mathrm{~nm}$.

\section{Result and Discussion}

\subsection{Polymer Synthesis and Characterization}

\subsubsection{Selection of the Polymerization Conditions}

Molecular imprinting could be carried out under a variety of different conditions with free radical reaction. For the common initiator AIBN, the homolysis reaction occurs by heating or irradiation at $365 \mathrm{~nm}$ using a mercury vapor lamp.

Generally, the polymerization was carried out with heat about $60^{\circ} \mathrm{C}$. In this article, heat polymerization and ultraviolet radiation at $365 \mathrm{~nm}$ were investigation. Polymer $1^{\#}$ and $2^{\#}$ were synthesized under the different polymerization condition. Figure 2 showed that the results showed that polymer $1^{\#}$ tended to form particles with smaller dimensions. Though polymer $1^{\#}$ had the better recognition efficiency, the difference between the MIP and NIP was inferior to the polymer of polymer $2^{\#}$ (Table 2). In the later experiments, ultraviolet radiation at $365 \mathrm{~nm}$ was selected as the polymerization mode.

\subsubsection{Selection of Functional Monomer}

Functional monomers strongly interact with the templatefunctional monomer complex prior to polymerization, since the solution structure of the resulting assemblies presumably defines the subsequently formed binding sites, therefore, it is of obvious importance to select the type of functional monomer. A typical functional mono-
Table 2. Porosities of molecularly imprinted polymers, as determined by BET analysis.

\begin{tabular}{cccc}
\hline Sample & $\begin{array}{c}\text { BET surface area } \\
\left(\mathrm{m}^{2} \cdot \mathrm{g}^{-1}\right)\end{array}$ & $\begin{array}{c}\text { Pore volume } \\
\left(\mathrm{cm}^{3} \cdot \mathrm{g}^{-1}\right)\end{array}$ & Pore size $(\AA)$ \\
\hline MIP & 23.0771 & 0.0892 & 128.88 \\
NIP & 27.5453 & 0.0888 & 104.83 \\
\hline
\end{tabular}

mer for noncovalent imprinting is methyacrylic acid (MAA), whose use was first proposed by Mosbach [21]. The broad applicability of MAA as a functional monomer is related to the fact that the carboxylic acid group serves well as a hydrogen bond and proton donor as well as a hydrogen bond acceptor. 4-Vinylpyridine (4-VP) is another functional monomer which is particularly well suited for the imprinting of carboxylic acid templates and provides materials with selectivity of the same order as those obtained using MAA for basic templates.

Polymer $2^{\#}$ and $5^{\#}$ were synthesized under the same conditions except for the functional monomers. The particle size of polymer $2^{\#}$ was about $2-3 \mu \mathrm{m}$, while polymer $5^{\#}$ was $300 \mathrm{~nm}$ with the heavy aggregation. Table 1 showed that polymer $2^{\#}$ had the better recognition efficiency than that of polymer $5^{\#}$. Therefore, $4-\mathrm{VP}$ was selected as functional monomer in the later experiments.

\subsubsection{Selection of Cross-Linker}

Another important factor in molecular imprinting is the type and quantity of the cross-linker, which are both critical for attaining high affinity. EGDMA is the most commonly used cross-linker for the methacrylate-based system, primarily because it provides materials with mechanical and thermal stability, good wettability in most rebinding media, and rapid mass transfer with good recognition properties [22]. Except for trimethacrylate monomers, such as TRIM can provide similar recognition properties for such a large variety of templates [23]. TRIM was used to prepare a molecularly imprinted solid- 

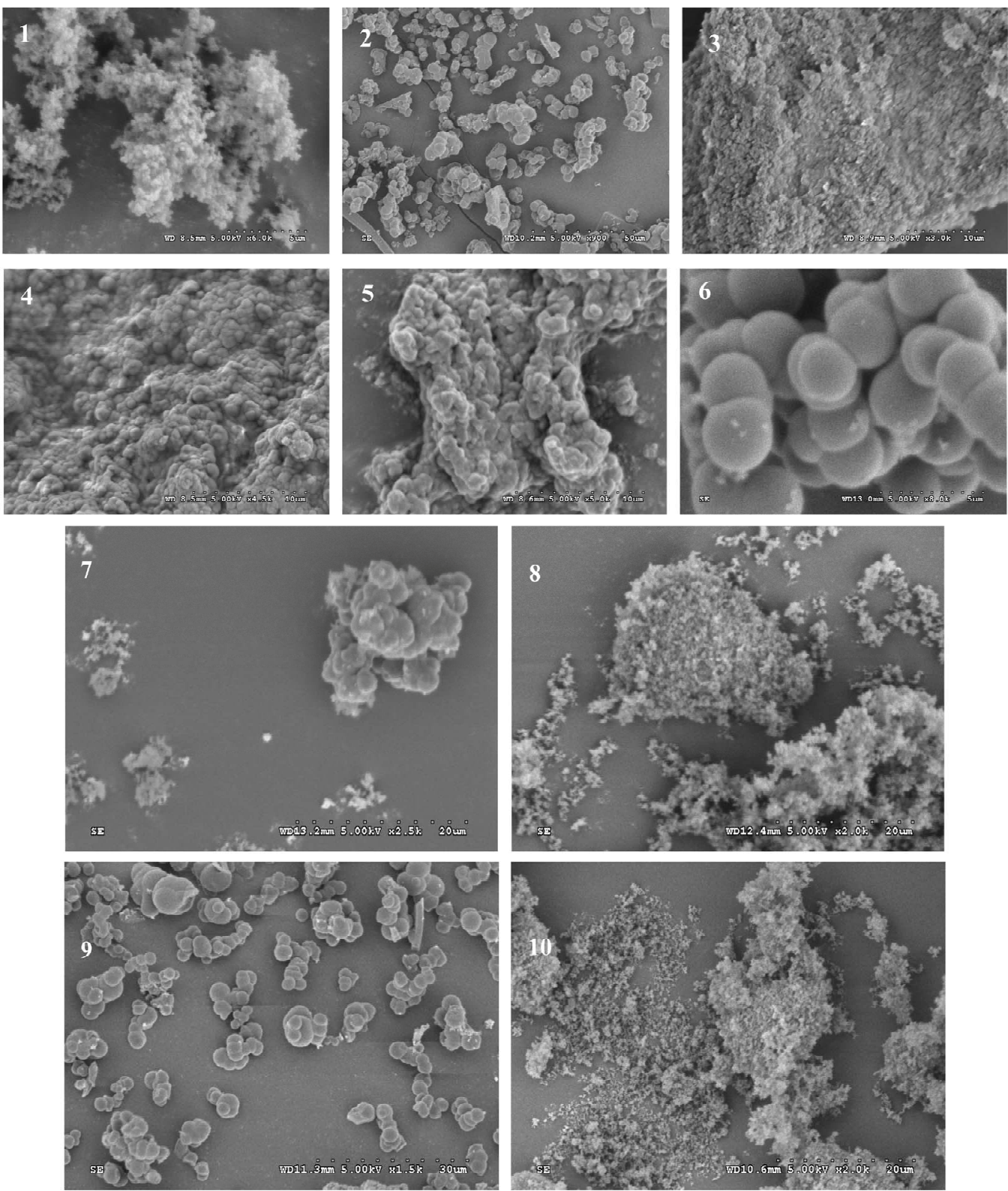

Figure 2. The scanning electron micrographs (SEM) of the suspersion polymers.

phase extraction (MISPE) sorbent possessing the highest binding selectivity and binding capability for the template tebuconazole [24]. DVB is also an alternative, e.g., Wei et al. [25] prepared a precipitation polymer with high binding selectivity by using DVB. In this study, several cross-linking monomers have been evaluated in molecular imprinting, such as EGDMA, DVB, and TRIM.

Polymer $2^{\#}, 3^{\#}$, and $4^{\#}$ were synthesized under the same polymerization conditions except for the type of crosslinkers. Figure 2 showed that since there was only considerably weak interaction between MAA and EGDMA (or TRIM), poly(MAA-co-EGDMA) or poly (MAA-co-TRIM) tended to form particles with smaller dimensions, while poly(MAA-co-DVB) tended toward particles of larger dimensions if the same ratio of template molecule, monomer to cross-linker was applied $(1: 4: 20)$. This was because that DVB had only one vinyl group to ensure the backbone rigidity. Table 1 showed that polymer $2^{\#}$ had a better recognition efficiency than that of polymer $3^{\#}$ and $4^{\#}$.

\subsubsection{Selection of Molar Ratio of Template Molecule and Functional Monomer to Cross-Linker}

Often, in the noncovalent imprinting system, a large excess of functional monomers in the synthesis step is required for recognition to be observed, moreover, to preserve the structural integrity of the monomer-template assemblies during the polymerization, a high level of 
cross-linker had to be ensured. In the traditional preparation method, the molar ratio of template molecule and functional monomer to crosslinker is 1:4:20 [26]. In this study, the molar ratios of $1: 4: 10,1: 4: 20$ and $1: 4: 30$ were chosen, respectively, to optimize the synthesized polymers.

As presented in Figure 2, polymers $9^{\#}$ and $2^{\#}$ were microspheres with the same diameter, while polymer $10^{\#}$ was submcrosphere. Table 1 showed that polymer $2^{\#}$ had better recognition efficiency than that of polymer $9^{\#}$ and $10^{\#}$. It might be concluded that the rebinding properties depent on the molar ratio, which may affect the template-functional monomer interactions.

\subsubsection{Selection of the Porogen}

The pivotal fact of this method is that the prepolymerization mixture should be insoluble in the mineral oil. The common solvents, such as chloroform, dichloromethane and toluene cannot be used as the porogen for this reason. We found, however, acetonitrile to work well. It is not soluble in mineral oil and has proven previously to be a good porogen of choice for the synthesis of MIPs [27,28]. Therefore, acetonitrile was selected as the porogen.

\subsubsection{The Influence of Amount of AIBN}

The double bonds in most vinylidene compounds of the general structure $\mathrm{CH}_{2}=\mathrm{CR}_{1} \mathrm{R}_{2}$ are reactive towards free radicals, cations or anions, forming transient species with a life-long enough to add to another double bond containing molecule. This generates a new active centre and repetition of this addition process leads to the formation of polymer. Due to its electrical neutrality, the free radical is a less selective and generally more useful initiator, this is one of the reasons why these system are particularly useful in molecular imprinting. For azo-initiators the free radicals are generated either by UV radiation at the wavelength for maximum absorption or thermally at a temperature providing a suitable rate of decomposition. In this study, free radical polymerization was chosen using AIBN as initiator. In the imprinting protocols developed thus far, the amount of AIBN added is based on the total monomer. 20, 60, 100, $160 \mathrm{mg}$ AIBN were selected, respectively, to optimize the amount of AIBN. It was found in Figure 2 that when the amount of AIBN was $100 \mathrm{mg}$, the polymer had the microsphere particles with better affinity efficiency.

\subsection{Evaluated Suspension Imprinted Polymer}

The binding assays were carried out as described in the Experimental Section of 2.4 and the experiment was done in water solution instead of acetonitrile since the determination of 2,4,6-TCP was applied in water samples. The experiment was performed in triplicate. The data obtained from batch-type method with 2,4,6-TCP con- centration from 16 to $162 \mathrm{mg} \cdot \mathrm{L}^{-1}$ were plotted according to the binding isotherm and shown in Figure 3. The maximum amount $Q_{\max }$ was estimated to be 107.05 $\mathrm{mg} \cdot \mathrm{g}^{-1}$ for MIP and $71.08 \mathrm{mg} \cdot \mathrm{g}^{-1}$ for NIP. The static adsorption capacity of the 2,4,6-TCP imprinted polymers was more than that of non-imprinted polymers. The results showed that the 2,4,6-TCP imprinted polymers had a higher adsorption capacity for 2,4,6-TCP. They would be more suitable to enrich trace $2,4,6-\mathrm{TCP}$ in water samples.

The porosities of the suspension polymers were determined by nitrogen adsorption/desorption analysis of Brennauer-Emmet-Teller (BET) multi-point adsorption isotherms. Table 2 showed that the pore volume and the specific surface area of the suspension polymers are similar to corresponding control materials synthesized in the absence of the template molecules. Therefore, any differential 2,4,6-TCP loadings displayed by the polymer was not caused by the morphological distinction but by the imprinting effect.

\subsection{Adsorption Kinetics on Imprinted Microspheres}

A rebinding kinetics study had been carried out for suspension polymer particles to determine the rate of the adsorption process. This is an essential consideration in the actual application of the material in the separation process. As shown in Figure 4, the MIP based on polymer adsorbed 2,4,6-TCP very quickly in the first $2 \mathrm{~h}$, after which the speed declined rapidly; after $6 \mathrm{~h}$, the MIP no longer adsorbed 2,4,6-TCP. This was the typical kinetics for most rebinding processes. During the initial rebinding process, the presence of a large amount of unoccupied, high-affinity binding sites on the surface of the particles enabled fast adsorption of 2,4,6-TCP molecules. When all the cavities on the surface were occupied, the template molecules should transport to the inner surface of the MIP which would take much more time. When most binding sites were filled up, the rate of adsorption dropped significantly and the 2,4,6-TCP loading was eventually achieved.

\subsection{Procedure of the MIMSPE}

The MIMSPE device consists of the MIPs enclosed within a polypropylene flat-sheet membrane envelope structure with the pore size of $0.22 \mu \mathrm{m}$ which can keep the sorbent in the sheet. The schematic of a typical MIMSPE device is shown in Figure 1. Each MIMSPE device was conditioned by ultrasonication in ultrapure water and acetonitrile for $2 \mathrm{~min}$. For extraction, two of the MIMSPE devices were placed in $60 \mathrm{~mL}$ aqueous sample and shaken for $6 \mathrm{~h}$. The device tumbled freely within the sample solution during extraction. After ex- 


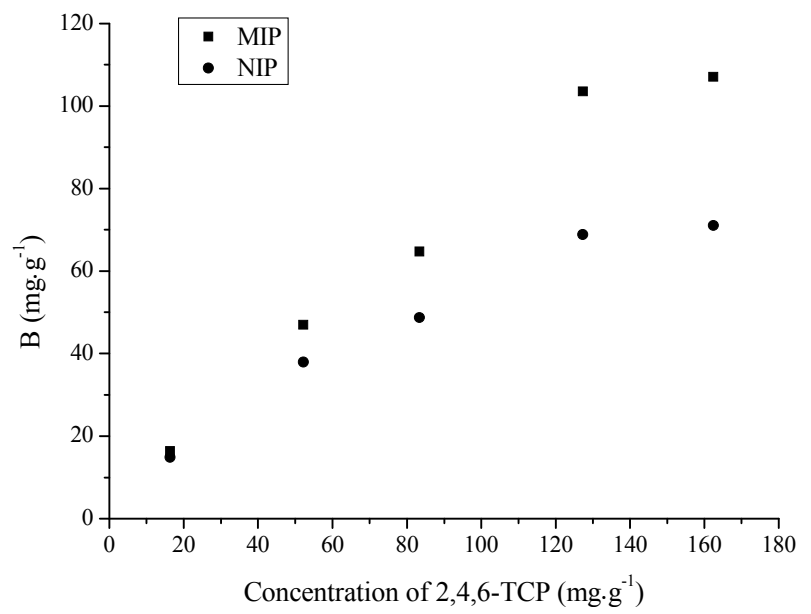

Figure 3. Binding isotherm of 2,4,6-TCP-imprinted polymer (MIP-2, NIP-2).

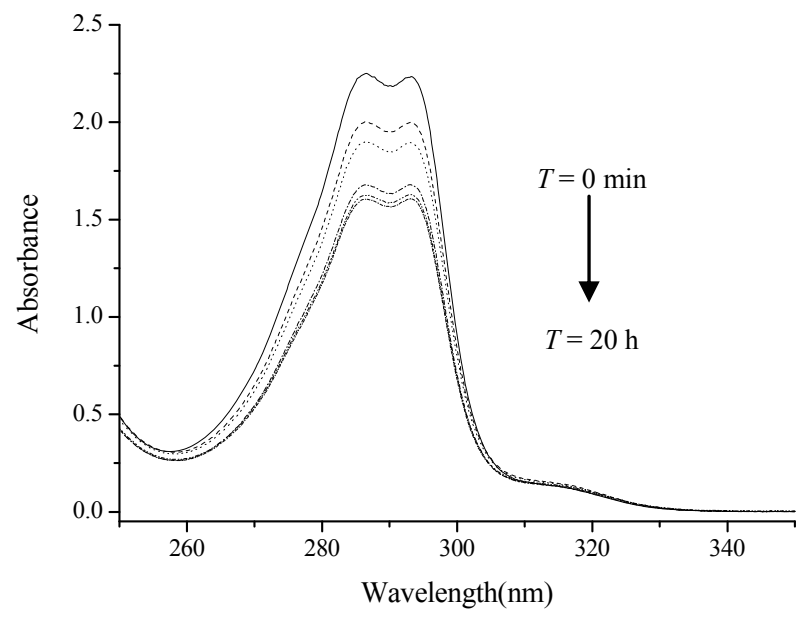

Figure 4. Adsorption kinetics of MIP (from top to bottom: $t$ $=0 \mathrm{~min}, 10 \mathrm{~min}, 30 \mathrm{~min}, 1 \mathrm{~h}, 2 \mathrm{~h}, 6 \mathrm{~h}, 20 \mathrm{~h}$ ).

traction, the device was removed, rinsed in ultrapure water, dried with filter paper, and placed in a sample vial. Tweezers were used to handle the device. The analytes were desorbed by ultrasonication in the solution of acetonitrile/acetic acid $(99.7: 0.3, \mathrm{v} / \mathrm{v}, 2.0 \mathrm{~mL}$ ) for $40 \mathrm{~min}$. The eluent was then purged with nitrogen to $0.5 \mathrm{~mL}$, out of which $20 \mu \mathrm{L}$ was used for HPLC analysis. The MIMSPE device could be reused after careful rinsing with acetonitrile.

\subsection{Determination of Phenolic Compounds with HPLC}

Determination of phenolic compounds (phenol, 4-CP, 2,4-DCP, 2,4,6-TCP and PCP) with HPLC was carried out as described in the Experimental Section. The mobile phase consisted of water, as solvent $\mathrm{A}$ and acetonitrile (containing $0.3 \%(\mathrm{v} / \mathrm{v})$ acetic acid) as solvent B. The flow rate of the mobile phase was $0.5 \mathrm{~mL} \cdot \mathrm{min}^{-1}$. The oven temperature was set at $40^{\circ} \mathrm{C}$, the injection volume was $20 \mu \mathrm{L}$, and all compounds were detected at $294 \mathrm{~nm}$, except for phenol and 4-CP which were detected at 280 $\mathrm{nm}$.

The method performance was evaluated by the determination of the linearity, sensitivity, repeatability, and accuracy of the method.

The linearity of the calibration curves were obtained by determination of the peak areas from analysis of spiked Mili-Q water at serial concentrations. All $r$-values were higher than 0.997 , the linear scopes were 0.05 $20.0 \mathrm{mg} \cdot \mathrm{L}^{-1}$.

The limits of detection (LOD), defined as the lowest analyte concentration with a signal-to-noise ratio of 3 , were investigated through the detection of spiked Mili-Q water at serial concentrations. The results showed that the LODs fell between 0.40 and $3.6 \mu \mathrm{g} \cdot \mathrm{L}^{-1}$, which indicated this method could be used to detect some polluted water samples.

\subsection{Analysis of Water Sample}

Water samples were collected in glass bottles. After the essential filtration treatment, the collected samples were stored in dark at $4^{\circ} \mathrm{C}$ and the further MIMSPE procedure was accomplished within $24 \mathrm{~h}$ to avoid any microbial degradation of analytes. Tap water samples were directly collected from the water faucet in our laboratory. River water samples were obtained from the Qinghe River in the northwest of Beijing. The raw sewage samples were collected from influent of sewage treatment plant (STP) which mainly receives domestic water from the uptown (population of $~ 8000$ ) located around the laboratory. Filtration is an essential step for real water samples to avoid matrix interference, even for the clean water such as tap water. In this study, freshly collected river water sample and tap water sample were filtered with $0.45 \mu \mathrm{m}$ filter while the sewage water was filtered with $0.20 \mu \mathrm{m}$ filter to remove suspended particles prior to extraction.

The recoveries obtained for phenol, 4-CP, 2,4-DCP, $2,4,6-\mathrm{TCP}, \mathrm{PCP}$ was in the range of $83.2 \%-104.8 \%$ for tap water, $82.0 \%-98.6 \%$ for river water and $78.4 \%$ $93.9 \%$ for sewage water. The RSD values of targets were lower than $10 \%$ which were also excellent. It demonstrated that 2,4,6-TCP MIPs had a satisfactory performance for the enrichment of phenolic compounds.

The MIMSPE-HPLC method established in this study was applied to quantitatively measure the phenolic compounds in environmental waters. None of phenolic compounds could be determined in nonspiked water (tap water, river water or the raw sewage water), maybe because there was little phenolic compounds in the water samples in Beijing. For the spiked water sample $\left(0.8 \mathrm{mg} \cdot \mathrm{L}^{-1}\right)$, the concentration factor was 120 . The river matrix and the sewage matrix (in Figure 5) did not significantly interfere with detection. 


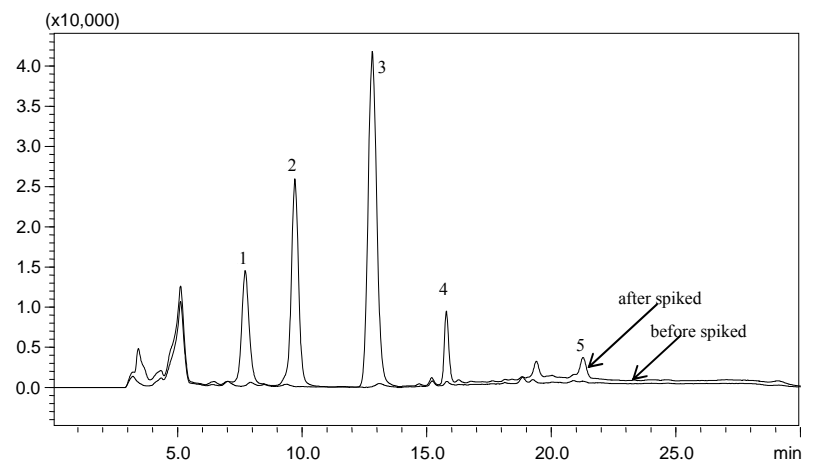

Figure 5. Chromatograms of sewage water sample before (upper line) and after (bottom line) spiked at $0.4 \mathrm{mg} \cdot \mathrm{L}^{-1}$ of each analyte. Peak identifications: (1): phenol, (2): 4-CP, (3): 2,4-DCP, (4): 2,4,6-TCP, (5): PCP.

\section{Conclusion}

The 2,4,6-TCP imprinted polymer was prepared by suspension polymerization using MAA and DVB as the monomer and cross-linker, acetonitrile as the porogen, ultraviolet radiation at $365 \mathrm{~nm}$, respectively. The obtained polymer showed good selectivity and enrichment efficiency with monodisperse spherical particles. Thus, a method was successfully developed by using the MAAco-DVB polymer as the MIMSPE coupled with HPLC for enrichment and analysis of phenolic compounds in environmental water. The high recoveries $(78.4 \%$ $104.8 \%)$ and satisfied precision $(0.8 \%-10.0 \%)$ for all the analytes proved that the method was valid for the analysis of some phenolic compounds mentioned above in different matrixes (tap water, river water, sewage water).

\section{Acknowledgements}

The authors gratefully acknowledge financial support of the National High-Tech Research and Development Program of China (863 program) (No. 2012AA062902) and the China Postdoctoral Science Foundation (grant Nos. 20090460520 and 201003155).

\section{REFERENCES}

[1] Z. H. Wang, X. L. Liu, J. M. Yang, Y. X. Qin and X. Q. $\mathrm{Lu}$, "Copper(II) Determination by Using Carbon Paste Electrode Modified with Molecularly Imprinted Polymer," Electrochimica Acta, Vol. 58, 2011, pp. 750-756. doi:10.1016/j.electacta.2011.10.034

[2] B. J. Sellergren, "Imprinted Dispersion Polymers: A New Class of Easily Accessible Affinity Stationary Phases," Journal of Chromatography A, Vol. 673, No. 1, 1994, pp. 133-141. doi:10.1016/0021-9673(94)87066-7

[3] H. Sambe, K. Hoshina, R. Moaddel, I. W. Wainer and J. Haginaka, "Uniformly-Sized, Molecularly Imprinted Polymers for Nicotine by Precipitation Polymerization,"
Journal of Chromatography A, Vol. 1134, No. 1-2, 2006, pp. 88-94. doi:10.1016/j.chroma.2006.08.073

[4] N. Pérez, M. J. Whitcombe and E. N. Vulfson, "Surface Imprinting of Cholesterol on Submicrometer Core-Shell Emulsion Particles," Macromolecule, Vol. 34, No. 4, 2001, pp. 830-836. doi:10.1021/ma001079v

[5] B. Q. Liu, D. P. Tang, B. Zhang, X. H. Que, H. H. Yang and G. N. Chen, "Au(III)-Promoted Magnetic Molecularly Imprinted Polymer Nanospheres for Electrochemical Determination of Streptomycin Residues in Food," Biosensors and Bioelectronics, Vol. 41, 2013, pp. 551-556. doi:10.1016/j.bios.2012.09.021

[6] Z. K. Lin, W. J. Cheng, Y. Y. Li, Z. R. Liu, X. P. Chen and C. J. Huang, "A Novel Superparamagnetic Surface Molecularly Imprinted Nanoparticle Adopting Dummy Template: An Efficient Solid-Phase Extraction Adsorbent for Bisphenol A," Analytica Chimica Acta, Vol. 720, 2012, pp. 71-76. doi:10.1016/j.aca.2012.01.020

[7] O. Prucker, J. Rühe, "Mechanism of Radical Chain Polymerizations Initiated by Azo Compounds Covalently Bound to the Surface of Spherical Particles," Macromolecules, Vol. 31, No. 3, 1998, pp. 602-613. doi:10.1021/ma970661p

[8] H. Kempe and M. Kempe, "Novel Method for the Synthesis of Molecularly Imprinted Polymer Bead Libraries," Macromolecular Rapid Communications, Vol. 25, No. 1, 2004, pp. 315-320. doi:10.1002/marc.200300189

[9] F Svec and J. M. Frechet, "Continuous Rods of Macroporous Polymer as High-Performance Liquid Chromatography Separation Media," Analytical Chemistry, Vol. 64, No. 7, 1992, pp. 820-822. doi:10.1021/ac00031a022

[10] J. Haginaka, "Monodispersed, Molecularly Imprinted Polymers as Affinity-Based Chromatography Media," Journal of Chromatography B, Vol. 866, No. 1-2, 2008, pp. 3-13. doi:10.1016/j.jchromb.2007.07.019

[11] A. G. Mayes and K. Mosbach, "Molecularly Imprinted Polymer Beads: Suspension Polymerization Using a Liquid Perfluorocarbon as the Dispersing Phase," Analytical Chemistry, Vol. 68, No. 21. 1996, pp. 3769-3774. doi:10.1021/ac960363a

[12] D. W. Zhu, "Perfluorocarbon Fluids: Universal Suspension Polymerization Media," Macromolecules, Vol. 29, No. 8, 1996, pp. 2813-2817. doi:10.1021/ma951540x

[13] H. Kempe, M. Kempe, "Development and Evaluation of Spherical Molecularly Imprinted Polymer Beads," Analytical Chemistry, Vol. 78, No. 11, 2006, pp. 3659-3666. doi:10.1021/ac060068i

[14] M. Zourob, S. Mohr, A.G. Mayes, A. Macaskill, N. Pérez-Moral, P. R. Fielden and N. J. Goddard, "A MicroReactor for Preparing Uniform Molecularly Imprinted Polymer Beads," Lab on a Chip, Vol. 6, No. 2, 2006, pp. 296-301. doi:10.1039/B513195b

[15] Z. G. Pei, X. Q. Shan, T. Liu, Y. N. Xie, B. Wen, S. Zhang and S. U. Khan, "Effect of Lead on the Sorption of 2,4,6-Trichlorophenol on Soil and Peat," Environmental Pollution, Vol. 147, No. 3, 2007, pp. 764-770. doi:10.1016/j.envpol.2006.09.001

[16] B. H. Hameed, "Equilibrium and Kinetics Studies of 2,4,6-Trichlorophenol Adsorption onto Activated Clay," 
Colloids and Surfaces A, Vol. 307, No. 1-3, 2007, pp. 4552. doi:10.1016/j.colsurfa.2007.05.002

[17] C. Basheer, A. A. Ainedhary, B. S. M. Rao, S. Valliyaveettil and H. K. Lee, "Development and Application of Porous Membrane-Protected Carbon Nanotube MicroSolid-Phase Extraction Combined with Gas Chromatography/Mass Spectrometry," Analytical Chemistry, Vol. 78, No. 8, 2006, pp. 2853-2858. doi:10.1021/ac060240i

[18] C. Basheer, H. G. Chong, T. M. Hii and H. K. Lee, "Application of Porous Membrane-Protected Micro-SolidPhase Extraction Combined with HPLC for the Analysis of Acidic Drugs in Wastewater," Analytical Chemistry, Vol. 79, No. 17, 2007, pp. 6845-6850. doi:10.1021/ac070372r

[19] Y. Xiong, "Flow-Injection Chemiluminescence Sensor for Determination of Isoniazid in Urine Sample Based on Molecularly Imprinted Polymer," Spectrochimica Acta Part A, Vol. 66, No. 2, 2007, pp. 341-346. doi:10.1016/j.saa.2006.03.001

[20] Q. Z. Feng, L. X. Zhao and J. M. Lin, "Molecularly Imprinted Polymer as Micro-Solid Phase Extraction Combined with High Performance Liquid Chromatography to Determine Phenolic Compounds in Environmental Water Samples," Analytica Chimica Acta, Vol. 650, No. 1, 2009, pp. 70-76. doi:10.1016/j.aca.2009.04.016

[21] K. Mosbach, "Molecular Imprinting,"Trends in Biochemical Sciences, Vol. 19, No. 1, 1994, pp. 9-14. doi:10.1016/0968-0004(94)90166-x

[22] A. L. Jenkins and G. M. Murray, "Polymer-Based Lanthanide Luminescent Sensor for Detection of the Hydrolysis Product of the Nerve Agent Soman in Water,"
Analytical Chemistry, Vol. 71, No. 2, 1999, pp. 373-378.

[23] B. Sellergren, "Molecularly Imprinted Polymers," 1st Edition, University of Mainz, Mainz, 2001

[24] M. L. Hu, M. Jiang, P. Wang, S. R. Mei, Y. F. Lin, X. Z. $\mathrm{Hu}, \mathrm{Y}$. Shi, B. Lu and K. Dai, "Selective Solid-Phase Extraction of Tebuconazole in Biological and Environmental Samples Using Molecularly Imprinted Polymers,' Analytical and Bioanalytical Chemistry, Vol. 387, No. 3, 2007, pp. 1007-1016. doi:10.1007/s00216-006-1004-2

[25] S. T. Wei, A. Molinelli and B. Mizaikoff, "Molecularly Imprinted Micro and Nanospheres for the Selective Recognition of $17 \beta$-Estradiol," Biosensors and Bioelectronics, Vol. 211, No. 10, 2006, pp. 1943-1951. doi:10.1016/j.bios.2005.09.017

[26] E. Caro, R. M. Marce, P. A. G. Cormack, D. C. Sherrington and F. Borrull, "On-Line Solidphase Extraction with Molecularly Imprinted Polymers to Selectively Extract Substituted 4-Chlorophenols and 4-Nitrophenol from Water," Journal of Chromatography A, Vol. 995, No. 1-2, 2003, pp. 233-238. doi:10.1016/S0021-9673(03)00543-0

[27] J. A. Tarbin and M. Sharman, "Development of Molecularly Imprinted Phase for the Selective Retention of Stilbene-Type Estrogenic Compounds," Analytica Chimica Acta, Vol. 433, No. 1, 2001, pp. 71-79. doi:10.1016/S0003-2670(00)01373-8

[28] I. S. Chronakis, A. Jakob, H. Bengt and Y. Lei, "Encapsulation and Selective Recognition of Molecularly Imprinted Theophylline and $17 \beta$-Estradiol Nanoparticles within Electrospun Polymer Nanofibers," Langmuir, Vol. 22, No. 21, 2006, pp. 8960-8965. doi:10.1021/la0613880 